http://jmscr.igmpublication.org/home/ ISSN (e)-2347-176x ISSN (p) 2455-0450

crossref DOI: https://dx.doi.org/10.18535/jmscr/v9i12.08

Journal Of Medical Science And Clinical Research

\title{
Missed diagnosis of Acromegaly in a patient with Type 2 Diabetes Mellitus: A Case Report
}

\author{
Authors \\ Dr Suman Kumar Sahu', Dr Sanghamitra Sahoo ${ }^{2}$, Dr Bijay Ketan Das ${ }^{3}$ \\ ${ }^{1,2,3}$ Postgraduate Department of General Medicine, VIMSAR
}

\begin{abstract}
The early stages of acromegaly are characterized by slow and progressive acral overgrowth and coarse facial features without major systemic complications. In some acromegaly patients, diabetes mellitus is accompanied with insulin resistance. Here we report a case of 39 year old female presented with altered sensorium, provisionally diagnosed to be Type 2 DM with HHS. She had secondary amenorrhea for last 1 year. The patient had no acromegaly-related complaints. Laboratory data revealed high blood levels of glucose and HbAlc. Physical examination revealed features of acromegaly. MRI disclosed pituitary macroadenoma and laboratory investigation indicated a high blood concentration of growth hormone and Insulin like growth factor 1. The patient was diagnosed with Type 2 Diabetes mellitus with acromegaly, and acromegaly associated exacerbation of Diabetes. The patient was treated with insulin, somatostatin analogues (Octreotide) and Dopamine agonist (Cabergoline). The case highlights the importance of insulin resistance, hyperglycemia and abnormal manifestations of face and feet in patients with acromegaly. In addition, these findings highlight the importance of the early diagnosis of acromegaly for preventing the consequences of inappropriate patient care.

Keywords: Acromegaly, Type 2 DM.
\end{abstract}

\section{Introduction}

The most frequent cause of acromegaly is pituitary hyper- secretion of growth hormone (GH). High levels of GH and insulin-like growth factor-1 (IGF-1) result in systemic alterations such as acral enlargement, skin thickening, soft tissue hyperplasia and excessive sweating ${ }^{1}$. GH excess affects insulin sensitivity and gluconeogenesis and can alter pancreatic $\beta$-cell function, leading to a derangement of glucose metabolism in a considerable percentage of acromegaly patients ${ }^{2}$. Indeed, impaired glucose tolerance (IGT) or diabetes mellitus (DM) are considered a frequent and - in many cases - an early manifestation of acromegaly ${ }^{3}$. Acromegaly is a highly debilitating disease associated with severe comorbidities, including cardiovascular, metabolic, respiratory, neoplastic and musculoskeletal complications, which significantly impact on patients' quality of life and mortality risk. Overall mortality is increased about three folds and is due primarily to cardiovascular and cerebrovascular disorders and respiratory disease ${ }^{4}$. Unless GH levels are controlled, survival is reduced by an average of 10 years compared with and age matched control population ${ }^{4}$. Acromegaly-associated physical alterations may not be detected by the patients at the early stages of disease because of slow disease progression and limited symptoms. The present 
report outlines a case of acromegaly that was undiagnosed for many years.

\section{Case Presentation}

A 39 year old female patient presented to our hospital with complains of altered sensorium for 2 days and vomiting for 2 days. There was no history of fever, headache, convulsion or loss of consciousness. She had suffered from Type 2 DM for past 5 years and was on oral hypoglycemic agent. She had been unable to control blood sugar adequately and she was started on regular insulin. And she did not have subsequent medical follow up for last 7 months. No other relevant past medical history. On Menstrual history amenorrhea for 1year.

On admission, patient was stuporous, BP-70/50 mmhg, PR-124/min, SpO2-95\% with room air, capillary blood glucose-High $(>600 \mathrm{mg} / \mathrm{dl})$. She had frontal bossing, mandibular enlargement, prognathism, prominent supra ciliary arches, large fleshy nose, coarse facial features, large tongue and feet. Her thyroid gland was not enlarged. On CNS examination-Pt. was stuporous, B/L pupil equal in size reacting to light, signs of meningeal irritation absent, B/L plantar flexor. Rest of the system examination was normal.

With provisional diagnosis of Type $2 \mathrm{DM}$ with HHS, she was given $0.9 \%$ NS 2.5 L over 3 hours. She was also started on regular insulin 6 units iv bolus followed by 6 units per hour by continuous iv infusion. Her blood sugar was not adequately controlled, so insulin dose was doubled to 12 units per hour by continuous iv infusion. Then her blood sugar came to $210 \mathrm{mg} / \mathrm{dl}$ and she was started on subcutaneous long-acting insulin with 2 hours overlap with insulin infusion. And on Day 3 she improved her consciousness and she was started on Basal bolus regimen with regular insulin and Insulin Glargine.

Urine analysis revealed absence of ketone body, sugar-2 g\%, albumin-TRACE, RBC-NIL, Pus cells-NIL.

Her blood chemistry as follows-RBS- $625 \mathrm{mg} / \mathrm{dl}$, HbA1c-14.4 \%, Urea-48mg/dl, Creatinine-1.3 $\mathrm{mg} / \mathrm{dl}$, Protein-6.2 mg/dl, albumin-2.2 mg/dl, Sodium-147mmol/L, Potassium-3.3 $\mathrm{mmol} / \mathrm{L}$, Calcium (ionised)-1.1 mmol/L, Cholesterol-126 mg/dl, HDL-26 mg/dl,LDL-32 mg/dl,VLDL-57 $\mathrm{mg} / \mathrm{dl}$, Triglyceride- $285 \mathrm{mg} / \mathrm{dl}$, Total bilirubin$0.4 \mathrm{mg} / \mathrm{dl}$, direct bilirubin- $0.1 \mathrm{mg} / \mathrm{dl}$,AST-15 IU/L, ALT-10 IU/L,ALP-167 IU/L,TLC-18.38x 10^3/microL, Neutrophil-91.5\%,Lymphocyte$6.9 \%$, Monocyte- $1.6 \%$, Eosinophil-0\%, Basophil0\%, Hb-10g/dl,MCV-65.7fL,MCH-22pg,MCHC$34.4 \mathrm{~g} / \mathrm{dL}$, TPC-281X10^3/microL.

She was having features of acromegaly and secondary amenorrhea. So MRI brain was done. On MRI a 26x28x45mm sized (APXTVSXCC) avidly enhancing mass is seen involving the sellapituitary and extending cranially in suprasellar region, elevating optic chiasm, encasingcavernous sinus. S/O-mass lesion like Pituitary macroadenoma.
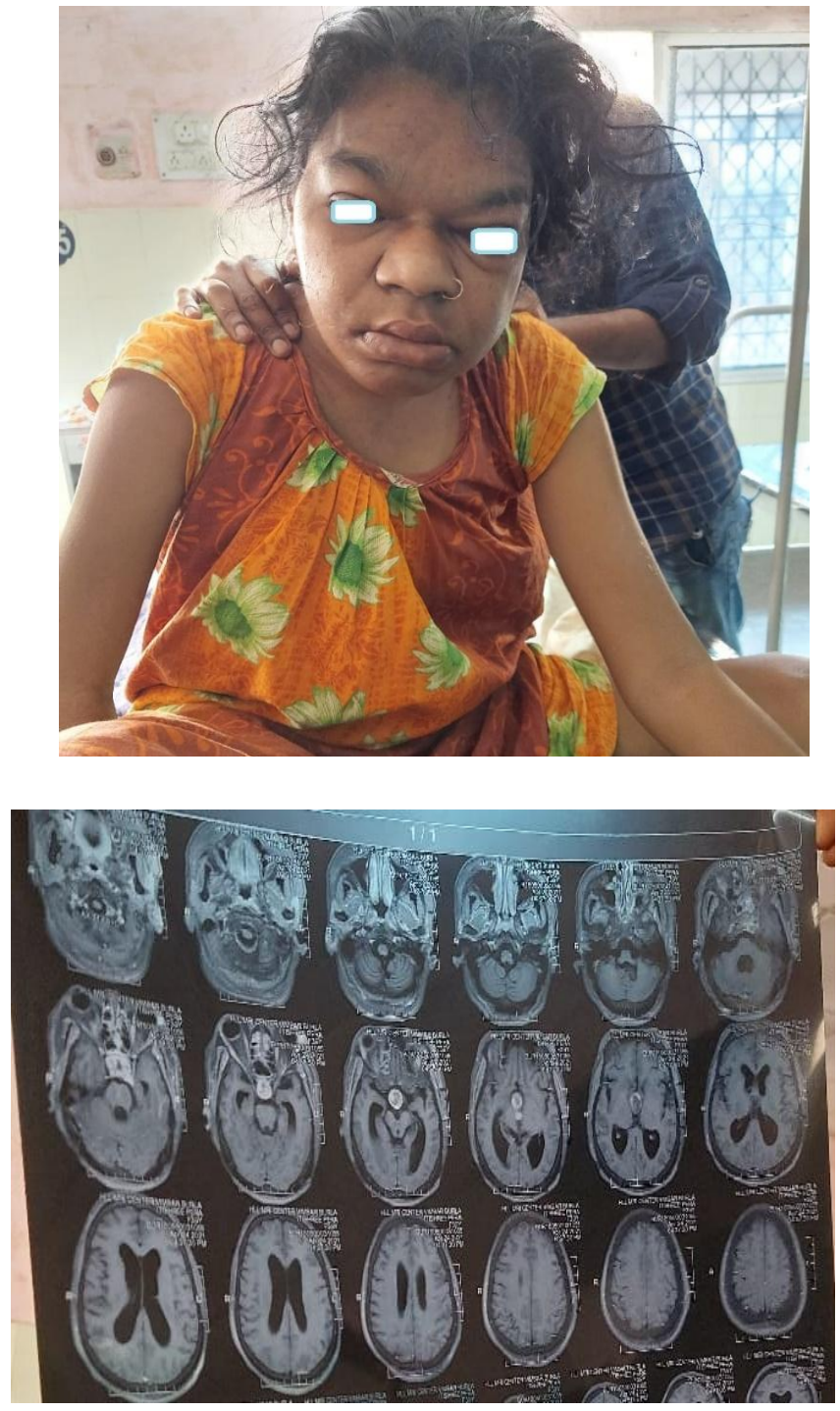


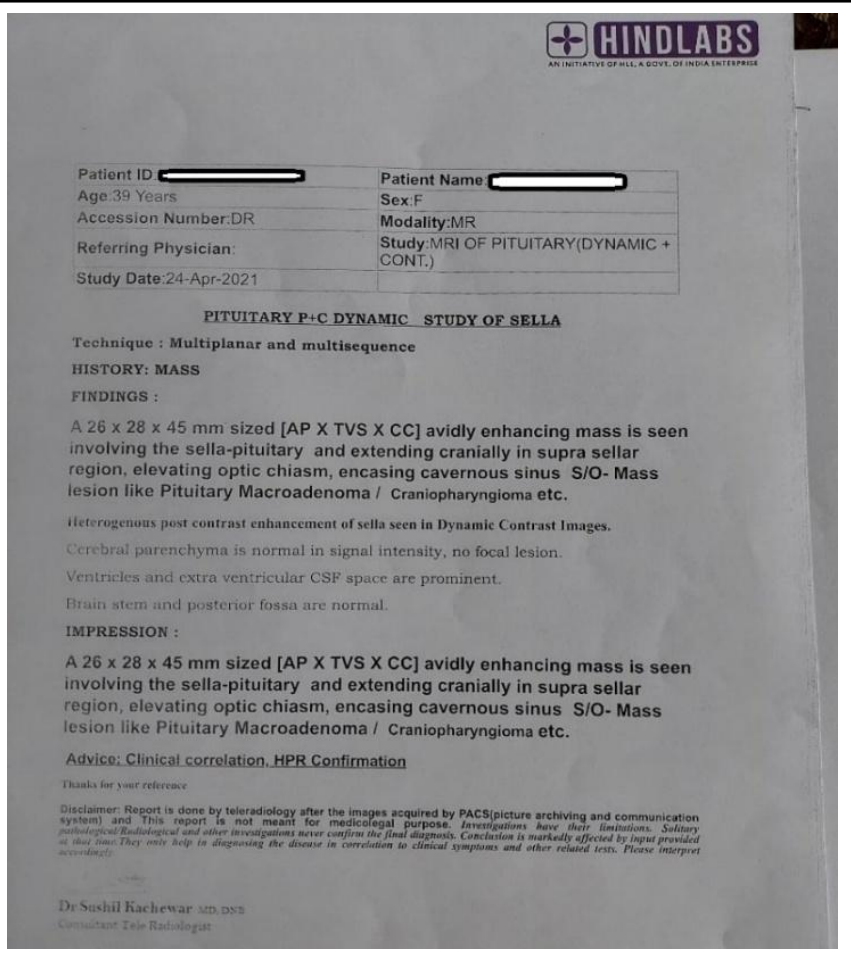

Endocrine Parameters

\begin{tabular}{|l|c|c|}
\hline Variable & value & Normal Range \\
\hline GH & 212 & $<1 \mathrm{ng} / \mathrm{ml}$ \\
\hline IGF1 & 454 & $92-255 \mathrm{ng} / \mathrm{ml}$ \\
\hline Prolactin & 1.40 & $3.34-26.72 \mathrm{ng} / \mathrm{ml}$ \\
\hline TSH & 0.53 & $0.5-5 \mathrm{miu} / \mathrm{ml}$ \\
\hline FT3 & 3.11 & $1.82-5.4 \mathrm{pg} / \mathrm{ml}$ \\
\hline FT4 & 0.96 & $0.7-1.8 \mathrm{ng} / \mathrm{ml}$ \\
\hline HbA1C & 14.4 & $<6.5 \%$ \\
\hline
\end{tabular}

Ophthalmic examination revealed no vision loss secondary to pituitary tumor or diabetic retinopathy. There were no symptoms of Cushing's syndrome

The patient was diagnosed with Type 2 Diabetes mellitus with acromegaly, and acromegaly associated exacerbation of Diabetes. However, tumor removal will ultimately clarify whether type 2 diabetes mellitus is only secondary to acromegaly. She was started on Injection Octreotide 50 microgram tid and tablet Cabergoline $(0.5 \mathrm{mg})$ OD. Patient was referred to higher centre for surgical removal of tumor.

\section{Discussion}

Acromegaly is caused by excessive secretion of growth hormone $(\mathrm{GH})$ and resultant elevation of Insulin Like Growth factor 1 (IGF1). The delay in diagnosis may exacerbate complications due to
GH hypersecretion. Thus timely diagnosis of acromegaly is crucial. Acromegaly is associated with severe comorbidities, including cardiovascular, metabolic, respiratory, neoplastic and musculoskeletal complications, which significantly impact on patients' quality of life and mortality risk. Overall mortality is increased about three folds and is due primarily to cardiovascular and cerebrovascular disorders and respiratory disease ${ }^{4}$. The disorders of glucose metabolism, including impaired fasting glycaemia (IFG), IGT and DM, are more frequent in patients with acromegaly than in the general population even when considering the groups at higher risk of developing diabetes (24-28). The prevalence of IGT or IFG ranges from 6 to $45 \%$ and from 7 to $22 \%$, respectively, while DM has been reported in $16-56 \%$ of acromegaly patients ${ }^{5,6}$. The first therapeutic option for acromegaly is surgical resection of the pituitary adenoma, although surgery is not feasible in patients with large tumors ${ }^{6}$. Patients with unresectable tumors receive treatment with Somatostatin analogues (Octreotide, Lanreotide, Pasireotide)and GH receptor antagonists (Pegvisomant), Dopamine agonist (Bromocriptine, Cabergoline) ${ }^{6}$. However, subtle progressive physical alterations may go unnoticed by patients and attending physicians, leading to delayed or missed diagnoses with detrimental implications for patient care. The present case indicated that, in addition to diabetic foot, acral abnormalities (such as enlarged feet) should also be assessed during the routine examination of patients with uncontrolled diabetes mellitus with severe hyperglycemia. The present report is an illustrative case of missed diagnosis of acromegaly at the early stages and the patient could not undergo debulking surgery of the pituitary tumor. Another point that could have prevented a missed diagnosis in the present case is the detection of hyperglycemia. Diabetes mellitus is a metabolic disorder that may be associated with acromegaly. A cohort study in a Mexican population revealed that, among patients with acromegaly, the incidence of diabetes mellitus 
appeared to be higher in women than in men ${ }^{6}$. However, gender could have a role in determining metabolic alterations, since women with active untreated acromegaly showed higher basal insulin levels and HOMA-IR and lower insulin sensitivity, higher visceral adiposity dysfunction and more frequently features of metabolic syndrome than men, regardless of GH and IGF-1 values ${ }^{7}$. Moreover, acromegaly patients have been reported to develop DM at younger age as compared to general population ${ }^{8}$. Furthermore, it has been found that in a variable percentage of patients the diagnosis of DM predates that one of acromegaly. In the present case, improvement of glucose metabolism after surgical treatment may demonstrate that acromegaly was the cause of hyperglycemia. The patient will undergo surgery when the tumor becomes operable. The current report is a typical case of acromegaly which was missed over a number of years, the most appropriate treatment was not received. The present report outlines a case of missed diagnosis of acromegaly associated with severe hyperglycemia. The associated pituitary tumor became macroadenoma in due course of time. This case highlights the importance of hyperglycemia in acromegaly and the critical importance of early diagnosis of acromegaly to prevent devastating consequences for patient care.

\section{Conclusion}

Here we report a case of missed diagnosis of acromegaly in aType 2 Diabetes mellitus patient where acromegaly exacerbated diabetes. In the present case, improvement of glucose metabolism after surgical treatment will demonstrate that acromegaly was the cause of hyperglycemia but she was lost to follow up.

Conflict of Interest: No

\section{References}

1. Colao A, Grasso LFS, Giustina A, Melmed S, Chanson P, Pereira AM and Pivonello
R: Acromegaly. Nat Rev Dis Primers 5: 20, 2019.

2. Frara S, Maffezzoni F, Mazziotti G, Giustina A. Current and emerging aspects of diabetes mellitus in acromegaly. Trends Endocrinol Metab. (2016) 27:470-83. doi: 10.1016/j.tem.2016.04.014

3. Colao A, Ferone D, Marzullo P, Lombardi G. Systemic complications of acromegaly: epidemiology, pathogenesis, and management. Endocr Rev. (2004) 25:10252. doi: 10.1210/er.2002-0022

4. Jameson J, \& Fauci A.S., \& Kasper D.L., \& Hauser S.L., \& Longo D.L., \&Loscalzo J(Eds.), (2018). Harrison's Principles of Internal Medicine, 20e. McGraw Hill.

5. Alexopoulou O, Bex M, Kamenicky P, Mvoula AB, Chanson P, Maiter D. Prevalence and risk factors of impaired glucose tolerance and diabetes mellitus at diagnosis of acromegaly: a study in 148 patients. Pituitary (2014) 17:81-9. doi: 10.1007/s11102-013-0471-7

6. Ferraù F, Albani A, Ciresi A, Giordano C, Cannavò S. Diabetes Secondary to Acromegaly: Physiopathology, Clinical Features and Effects of Treatment. Front Endocrinol (Lausanne). 2018 Jul 6;9:358. doi: 10.3389/fendo.2018.00358. PMID: 30034367; PMCID: PMC6043782

7. Ciresi A, Amato MC, Pivonello R, Nazzari E, Grasso LF, Minuto F, et al. The metabolic profile in active acromegaly is gender-specific. J Clin Endocrinol Metab. (2013) 98:E51-9. doi: 10.1210/jc.20122896

8. Arosio M, Reimondo G, Malchiodi E, Berchialla P, Borraccino A, De Marinis L, et al. Predictors of morbidity and mortality in acromegaly: an Italian survey. Eur J Endocrinol. (2012) 167:189-98. doi: 10.1530/EJE-12-0084. 